\title{
EVIDENCE FOR MAGNETIC FLUX SATURATION IN RAPIDLY ROTATING M STARS
}

\author{
A. REINERs ${ }^{1,3}$, G. BASRI ${ }^{2}$, AND M. BROWNING ${ }^{2,4}$ \\ ${ }^{1}$ Institut für Astrophysik, Georg-August-Universität, D-37077 Göttingen, Germany; Ansgar.Reiners@phys.uni-goettingen.de \\ ${ }^{2}$ Astronomy Department, University of California, Berkeley, CA 94720, USA; basri@berkeley.edu, mbrowning@berkeley.edu \\ Received 2008 July 4; accepted 2008 October 28; published 2009 February 19
}

\begin{abstract}
We present magnetic flux measurements in seven rapidly rotating $\mathbf{M}$ dwarfs. Our sample stars have X-ray and $\mathrm{H} \alpha$ emission indicative of saturated emission, i.e., emission at a high level, independent of rotation rate. Our measurements are made using near-infrared FeH molecular spectra observed with the High Resolution Echelle Spectrometer at Keck. Because of their large convective overturn times, the rotation velocity of M stars with small Rossby numbers is relatively slow and does not hamper the measurement of Zeeman splitting. The Rossby numbers of our sample stars are as small as 0.01 . All our sample stars exhibit magnetic flux of $\mathrm{kG}$ strength. We find that the magnetic flux saturates in the same regime as saturation of coronal and chromospheric emission, at a critical Rossby number of around 0.1 . The filling factors of both field and emission are near unity by then. We conclude that the strength of surface magnetic fields remains independent of rotation rate below that; making the Rossby number yet smaller by a factor of 10 has little effect. These saturated M-star dynamos generate an integrated magnetic flux of roughly $3 \mathrm{kG}$, with a scatter of about $1 \mathrm{kG}$. The relation between emission and flux also has substantial scatter.
\end{abstract}

Key words: stars: activity - stars: late-type - stars: magnetic fields - stars: rotation

Online-only material: color figures

\section{INTRODUCTION}

Stellar magnetic activity is closely related to rotation in solartype stars. In slow rotators, activity scales with the rotation rate until it becomes saturated at a certain velocity, which means that it does not grow further regardless of rotation rate (Noyes et al. 1984; Pizzolato et al. 2003). This effect is seen in a broad variety of activity indicators (Vilhu 1984, 1987). The threshold rate at which saturation occurs depends on the spectral type of the star, with the convective overturning time perhaps determining this threshold. Saturation sets in where the value of the Rossby number Ro $=P / \tau_{\text {conv }} \lesssim 0.1$, i.e., where the timescale of rotation is significantly shorter than the timescale of typical convective eddies.

Indicators of stellar activity are usually coronal or chromospheric emission observed at X-ray, UV, optical, IR, or radio wavelengths. We know from the Sun that this emission is induced by magnetic fields heating the upper layers of the solar atmosphere, and by analogy we conclude that stellar activity is connected to magnetic fields on the surface of other stars. At high rotation rates (or small Rossby numbers), all activity indicators saturate, i.e., they do not grow over a certain level, regardless of higher rotation rates (see, e.g., James et al. 2000). Two possible explanations exist for the saturation: (1) the stellar dynamo process saturates and stars cannot produce magnetic fields stronger than the saturation value; or (2) the magnetic fields continue to grow at more rapid rotation, but the fraction of the surface filled with fields - or the area covered by spots-reaches unity so that no more emitting plasma can be placed on the star. The only way to decide which way the stars go is to directly measure the magnetic field. Unfortunately, this is very difficult and the picture - particularly in stars with saturated activity—is not yet clear.

\footnotetext{
3 Emmy Noether Fellow.

4 Present address: Department of Astronomy and Astrophysics, University of Chicago, Chicago, IL 60637, USA.
}

Saar (1996) has collected measurements of magnetic flux $B f$, of the filling factor $f$, and of rotation periods to investigate the behavior of magnetic fields on stars. It is important to realize that the magnetic flux, or the magnetic field average over the whole surface, is not the same as the local field. It is the (unsigned) mean average of the magnetic field strength over the whole surface. Furthermore, the strongest magnetic fields in cool spots may not be fully captured because their contribution to the total flux is diminished due to their low temperature. As on the Sun, magnetic flux is probably concentrated in relatively small regions of strong fields (see, e.g., Johns-Krull \& Valenti 2000). Saar (1996) shows that in stars rotating slower than the saturation threshold the magnetic flux $B f$ as well as the filling factor $f$ show the same trend as all other activity indicators-they grow with larger rotation rate. At high rotation rates, Saar (1996) claims that saturation occurs in the filling factor $f$ but not in the magnetic flux $B f$. From this result, one would conclude that a star rotating at the saturation threshold is completely covered with magnetism $(f=1)$, and that the saturation phenomenon is due to the saturation of the emission process, while the star's magnetic flux can grow further with higher rotation. Saar (2001) has reinvestigated this issue with a few more data points, noting that there is some indication for a saturation at $B f \sim 3 \mathrm{kG}$ at small Rossby numbers. Much higher magnetic flux could be in contradiction to the idea that magnetic fields in stellar atmospheres cannot grow stronger than the equipartition field, i.e., the field strength at which the magnetic pressure equals the gas pressure. However, Solanki (1994) shows that the equipartition field may not necessarily be a hard upper limit for the field strength at $f=1$, so that more rapidly rotating stars could in principle have much stronger fields.

The only way to decide whether $B f$ does saturate or not is to provide direct measurements of magnetic flux in the regime of saturated activity. Generally, the measurement of magnetic fields relies on the splitting of spectral lines through the Zeeman effect (e.g., Robinson 1980). In rapid rotators, the subtle effect of magnetic broadening is buried under the rotational line 
broadening, so that it is particularly difficult to directly measure the magnetic flux in the regime of saturated magnetic activity. However, the crucial datum for rotational line broadening is the projected rotation velocity $v \sin i$, and not the Rossby number or the rotation period (which are the numbers that appear to set the saturation threshold of magnetic activity). Mainly because of the smaller radius of cooler stars, the surface rotation velocity at which saturation sets in depends on spectral type. In early G-type stars, the saturation velocity in the transition region is of the order of $30 \mathrm{~km} \mathrm{~s}^{-1}$ (and only $15 \mathrm{~km} \mathrm{~s}^{-1}$ in the corona; Ayres 1999). In M dwarfs, it is less than $5 \mathrm{~km} \mathrm{~s}^{-1}$ (see, e.g., Reiners 2007). Thus, in Sun-like stars, the high surface rotation velocity required for activity saturation hampers the measurement of magnetic flux, but this regime can easily be probed in M stars.

One potential problem with the use of $M$ dwarfs for the investigation of dynamo related phenomena is that the interior structure changes around spectral type M3.5-stars cooler than that are completely convective. Nevertheless, no change in activity is observed at the threshold to complete convection. A rotation-activity connection is observed in $\mathrm{M}$ stars down to spectral types M8.5 (Mohanty \& Basri 2003; Reiners 2007).

In this paper, we present direct measurements of magnetic flux in several $M$ stars. Some of them are very rapid rotators and clearly belong to the regime of saturated activity. We aim to clarify whether the magnetic flux $B f$ saturates as $\mathrm{H} \alpha$ and $\mathrm{X}$-ray emission do, or if $B f$ continues to grow beyond $B f \sim 3 \mathrm{kG}$.

\section{SAMPLE AND OBSERVATIONS}

For our sample we chose a number of mid-M stars with known X-ray emission and presumably high rotation rates. The values $v \sin i$ and $\log L_{\mathrm{X}} / L_{\mathrm{bol}}$ are taken from Delfosse et al. (1998), except for GJ 3379. This value is calculated from the Xray luminosity taken from Schmitt \& Liefke (2004). With one exception, projected rotation velocities $v \sin i$ were available for all targets. We chose only stars in which $v \sin i$ was reported to be above $5 \mathrm{~km} \mathrm{~s}^{-1}$ and which show saturated normalized X-ray emission. For GJ 3379, we are not aware of any former $v \sin i$ measurement, but the high value of normalized $\mathrm{X}$-ray emission is indicative of saturation and we added the star to our sample.

Data were taken at the W.M. Keck Observatory with the High Resolution Echelle Spectrometer (HIRES). Our setup covers the wavelength range from below $\mathrm{H} \alpha(6560 \AA)$ up to the molecular absorption band of $\mathrm{FeH}$ around $1 \mu \mathrm{m}$. We used a slit width of $1.15^{\prime \prime}$ achieving a resolving power of about $R=31000$. Our $\log$ of observations is given in Table 1. Data were cosmic-ray corrected, flat-fielded, background-subtracted, and wavelengthcalibrated using a ThAr spectrum. Data reduction was carried out using routines from the echelle package within the European Southern Observatory / Munich Image Data Analysis system (ESO/MIDAS) distribution. Fringing is not an issue in spectra taken with the new HIRES CCD, even in very red spectral regions around $1 \mu \mathrm{m}$.

\section{ANALYSIS}

The analysis of our spectra follows the strategy laid out in Reiners \& Basri (2006, 2007; RB07 in the following). We measure the equivalent width of the $\mathrm{H} \alpha$ emission and convert this number to normalized $\mathrm{H} \alpha$ luminosity using M-star atmospheres calculated with the PHOENIX code (Allard et al. 2001). To measure the projected rotation velocity $v \sin i$ and the
Table 1

Log of Observations

\begin{tabular}{lccc}
\hline \hline Name & UTC Date & Exp. Time (s) & $\log L_{\mathrm{X}} / L_{\text {bol }}$ \\
\hline GJ 3379 & 2007 Sep 30 & 200 & -2.86 \\
GJ 2069 B & 2008 Jan 24 & 600 & -2.77 \\
Gl 493.1 & 2007 Apr 25 & 600 & -3.31 \\
LHS 3376 & 2007 Apr 25 & 1800 & -3.63 \\
GJ 1154 A & 2007 Apr 25 & 600 & -3.28 \\
GJ 1156 & 2007 Apr 25 & 600 & -3.39 \\
Gl 412 B & 2007 Apr 25 & 1200 & -3.28 \\
\hline
\end{tabular}

magnetic flux $B f$ of our sample stars, we utilize the absorption band of molecular $\mathrm{FeH}$ close to $1 \mu \mathrm{m}$. We compare our data with spectra of the slowly rotating M stars GJ 1002 (M5.5) and $\mathrm{G} 873^{5}$ (M3.5). To match the absorption strength of the target spectra, the intensity of the $\mathrm{FeH}$ absorption lines in the two comparison spectra is modified according to an optical-depth scaling (see Reiners \& Basri 2006). In a first step, we compare the artificially broadenend spectrum of GJ 1002 to the target spectra in the wavelength region at 9930-9960 A to determine the value of $v \sin i$ by $\chi^{2}$-minimization.

For the determination of the magnetic flux $B f$, we concentrate on smaller wavelength regions that contain absorption lines particularly useful for this purpose, i.e., regions that contain some magnetically sensitive as well as magnetically insensitive lines. The magnetic flux of Gl 873 was measured to be $3.9 \mathrm{kG}$ (using an atomic Fe I line; Johns-Krull \& Valenti 2000). For our measurement, we are using a spectrum that contains both the $\mathrm{Fe}$ I line and the $\mathrm{FeH}$ absorption band. The $\mathrm{Fe}$ I line in this spectrum is consistent with the same magnetic flux value as found by Johns-Krull \& Valenti (2000), so that we can use the $\mathrm{FeH}$ pattern for the calibration of magnetic flux measurements in other stars. This method does not require theoretical models of the magnetic Zeeman splitting of FeH lines, which are not available yet.

We determine the magnetic flux of our target stars by comparison of the spectral regions at 9895.5-9905.5, 9937.59941.0, 9946.0-9956.0, and 9971.5-9981.0 ^ (for more details see RB07). In Figures 1-4 we show the data and the quality of our fit in the top panels. Note that, in rapid rotators, the difference between magnetic and nonmagnetic stars is not necessarily clearest at the exact location of magnetically sensitive lines. The blending of lines through rotation pronounces differences at wavelengths where the equivalent widths of lines differ the most between active and inactive stars.

For example, the two $\mathrm{FeH}$ lines at 9949.1 and $9951.7 \AA$ are magnetically not very sensitive (see Reiners \& Basri 2006, 2008), but it appears that the $9949.1 \AA$ line effectively gains a little in equivalent width. Thus, at rotation rates as high as shown in Figures 3 and 4, the region around $9949 \AA$ becomes the one of largest difference. The reasons for this are the magnetically sensitive features next to insensitive lines together with the effective gain in equivalent width, although such differences are not necessarily overt for the observer once Doppler broadening is introduced. On the other hand, it can also happen that at wavelengths where the unrotated spectra are quite different, rotational broadening averages in adjacent flux in such a way that the magnetic differences end up erasing

\footnotetext{
5 Gl 873 is rotating at $v \sin i \sim 3 \mathrm{~km} \mathrm{~s}^{-1}$ (RB07), not at a higher velocity as reported in Delfosse et al. (1998). The small (but detectable) rotation of Gl 873 does not affect our measurements.
} 

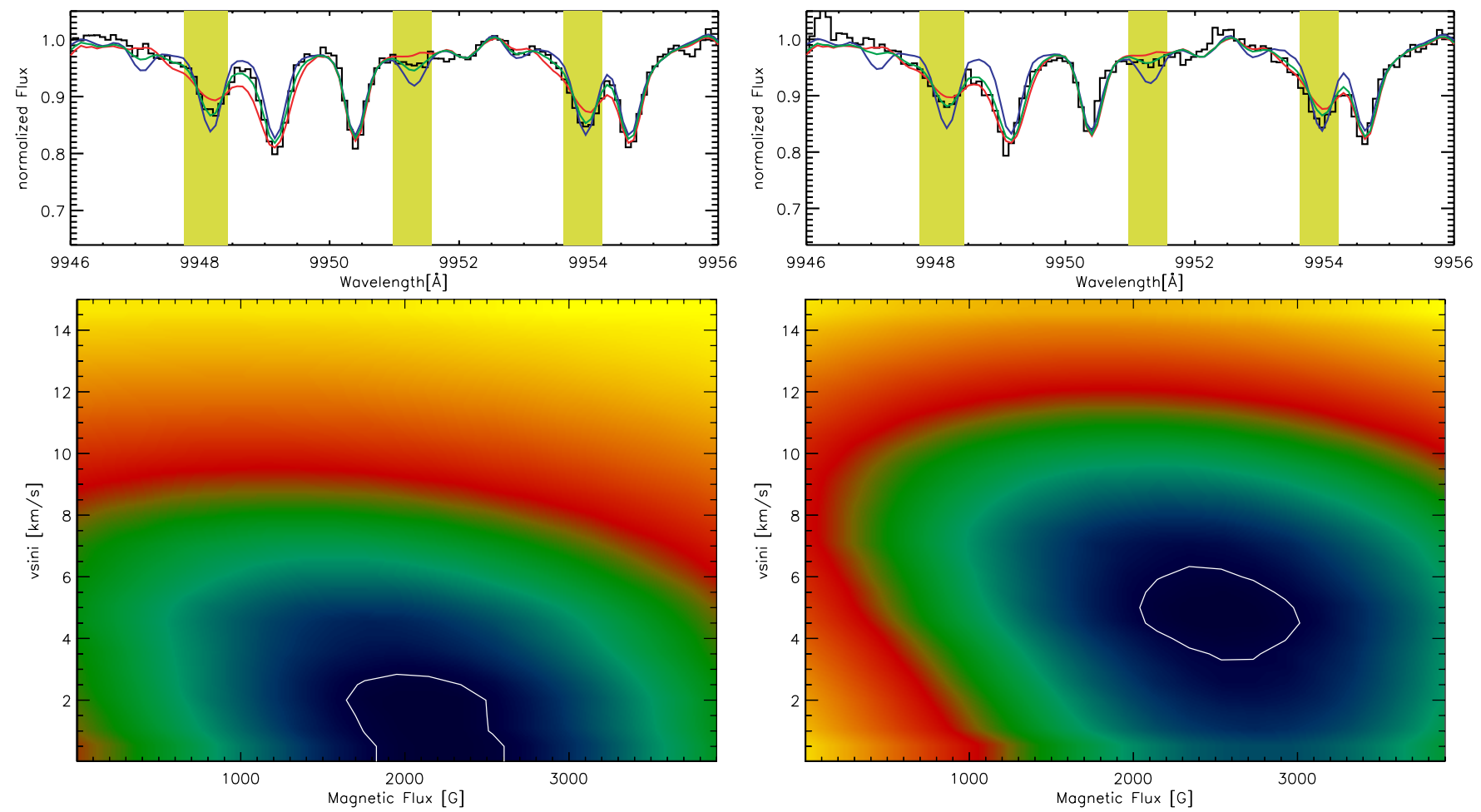

Figure 1. Top panel: data and fit of the two slow rotators GJ 3379 (left) and GJ 2069 B (right). The data are shown in black. Our fit to the data for the case of no magnetic flux is overplotted in blue, very strong magnetic flux $(B f=3.9 \mathrm{kG})$ in red, and the best fit with intermediate flux values in green. Bottom panel: $\chi^{2}$ landscapes showing the goodness of fit as a function of $v \sin i$ and $B f$. Dark/blue color indicates good fit quality, bright/yellow color means bad fit. The white contour marks the formal $3 \sigma$ region, i.e., $\chi^{2}<\chi_{\min }^{2}+9$.

(A color version of this figure is available in the online journal.)
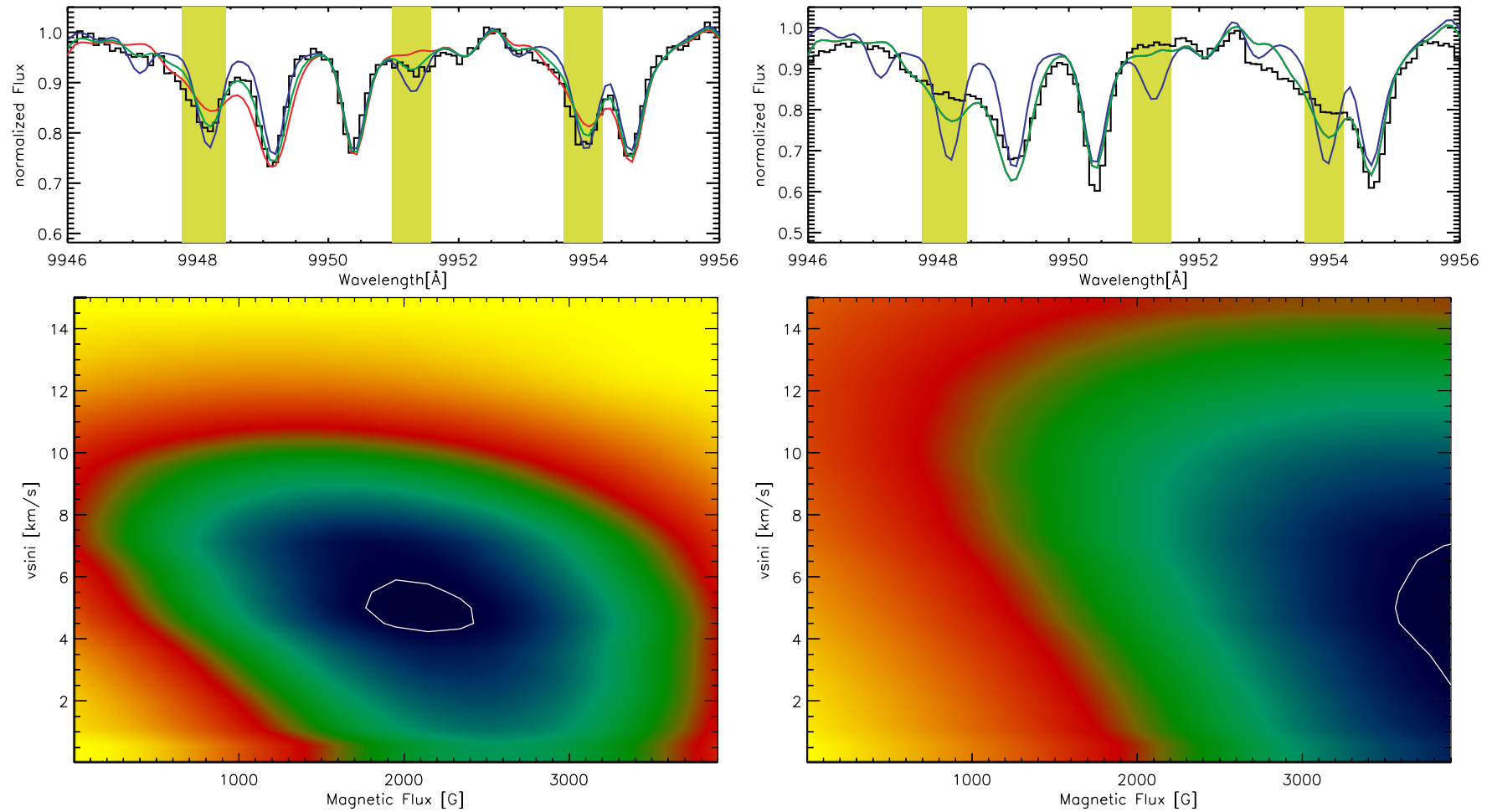

Figure 2. Date and fit (top panel) and $\chi^{2}$ landscapes (bottom panel) as in Figure 1 for the two intermediate rotators GJ 1154 A (left) and GJ 412 B (right). (A color version of this figure is available in the online journal.)

each other there (e.g., at $9948 \AA$ ). Thus, it is necessary to carry out a spectral fitting procedure after proper preparation instead of relying on appearances in original template spectra at fixed wavelengths. 

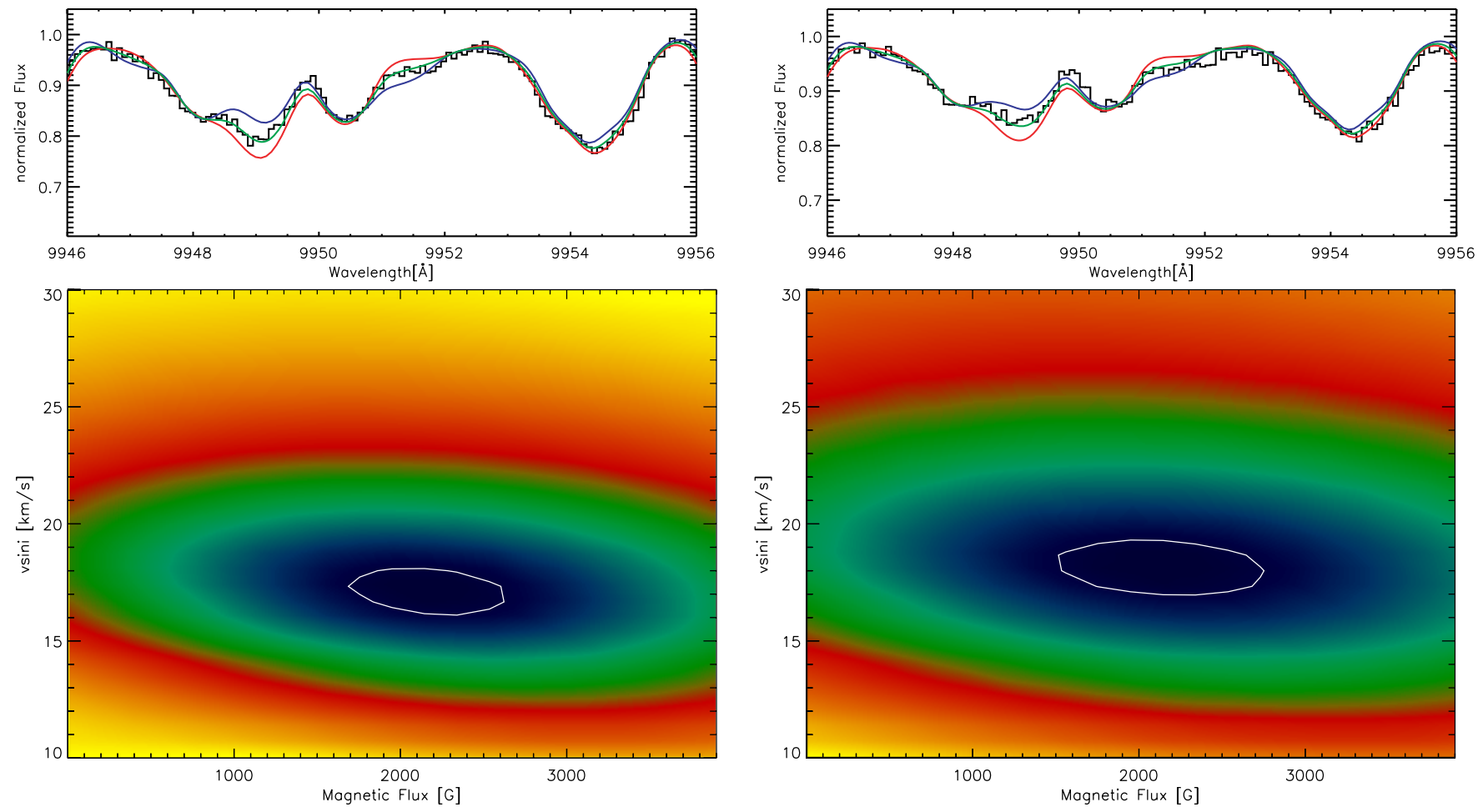

Figure 3. Data and fits (top panel) and $\chi^{2}$ landscape as in Figure 1 for the rapid rotators GJ 1156 (left) and G1 493.1 (right).

(A color version of this figure is available in the online journal.)
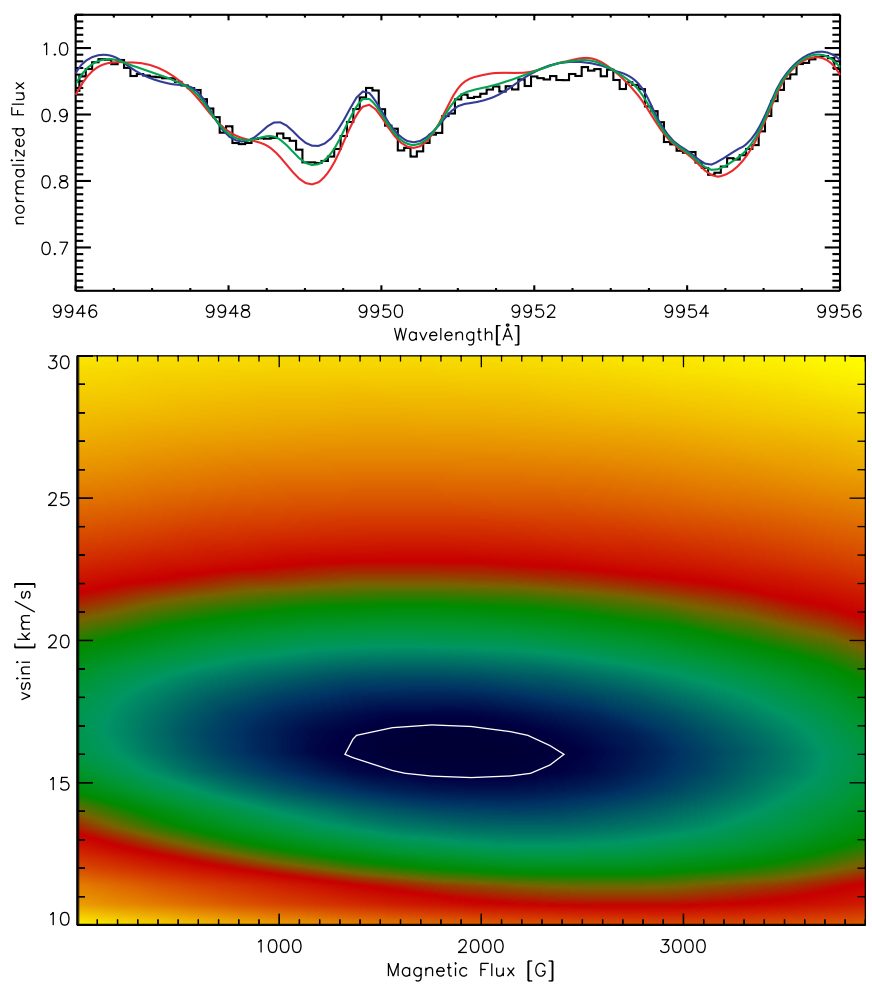

Figure 4. As Figure 3 for the rapid rotator LHS 3376.

(A color version of this figure is available in the online journal.)

\section{RESULTS}

In the bottom panels of Figures 1-4, we show the $\chi^{2}$ landscapes for all our targets as a function of $v \sin i$ and $B f$. Color coding displays the quality of the fit. In each $\chi^{2}$ landscape, the white contour marks the $3 \sigma$ region, i.e., $\chi^{2}<\chi_{\min }^{2}+9$ within this region. In all cases, the mean deviation per degree of freedom is of the order of $1\left(\chi_{v} \approx 1\right)$ for the estimated signal-tonoise ratio. Uncertainties in $v \sin i$ and $B f$ are typically around $1 \mathrm{~km} \mathrm{~s}^{-1}$ and a few hundred gauss, respectively. We emphasize that in particular in the case of $B f$ systematic errors are a more severe source of uncertainty so that the total uncertainty in $B f$ is more realistically in the $500-1000 \mathrm{G}$ range.

The results of our analysis are given in Table 2; projected rotation velocity $v \sin i$, magnetic flux or the mean magnetic field $B f$, and normalized $\mathrm{H} \alpha$ activity $\log L_{\mathrm{H} \alpha} / L_{\text {bol }}$ are given in Columns 3, 4, and 5, respectively. All $\mathrm{H} \alpha$ measurements confirm that our sample targets are active stars close to the activity saturation level. Three stars show very rapid rotation on the order of $20 \mathrm{~km} \mathrm{~s}^{-1}$; three stars are rotating at a velocity around $6 \mathrm{~km} \mathrm{~s}^{-1}$. GJ 3376 shows rotation below our detection limit of $v \sin i \approx 3 \mathrm{~km} \mathrm{~s}^{-1}$. For comparison, we include in Column 6 measurements of $v \sin i$ by Delfosse et al. (1998). In GJ 1156, we measure a rotational velocity three times higher than formerly reported, and in GJ 2069 B our new value of rotational broadening is $v \sin i=6 \mathrm{~km} \mathrm{~s}^{-1}$ while Delfosse et al. (1998) report $v \sin i=9 \mathrm{~km} \mathrm{~s}^{-1}$. We attribute the differences to higher data quality in our sample and to our more sophisticated fitting procedure. The uncertainty in $v \sin i$ also depends on the magnetic flux with a higher uncertainty at higher magnetic flux. The cross-talk between magnetic flux and rotation velocity may be weaker in other wavelength regions (but so far this has not been investigated in detail).

Measuring magnetic flux is hampered if a target is rapidly rotating because resolving individual lines becomes difficult in the presence of rotation. In the three rapid rotators Gl 493.1, LHS 3376, and GJ 1156, we used only the spectral regions 9946-9956 ̊ and 9972-9981 ̊, which are particularly well suited in rapid rotators (Reiners \& Basri 2006). 
Table 2

Results of Our Analysis

\begin{tabular}{lccccr}
\hline \hline Name & SpT & $\begin{array}{c}v \sin i \\
\left(\mathrm{~km} \mathrm{~s}^{-1}\right)\end{array}$ & $\begin{array}{l}B f \\
(\mathrm{G})\end{array}$ & $\log \frac{L_{\mathrm{H} \alpha}}{L_{\text {bol }}}$ & $\begin{array}{r}v \sin i_{\text {Lit }} \\
\left(\mathrm{km} \mathrm{s}^{-1}\right)\end{array}$ \\
\hline GJ 3379 & M3.5 & $>3$ & 2300 & -3.35 & \\
GJ 2069 B & M4.0 & 6 & 2700 & -3.28 & 9.2 \\
G1 493.1 & M4.5 & 18 & 2100 & -3.46 & 16.8 \\
LHS 3376 & M4.5 & 19 & 2000 & -3.73 & 14.6 \\
GJ 1154 A & M5.0 & 6 & 2100 & -3.55 & 5.2 \\
GJ 1156 & M5.0 & 17 & 2100 & -3.53 & 6.5 \\
G1 412 B & M6.0 & 5 & $>3900$ & -3.72 & 7.7 \\
\hline
\end{tabular}

Note. Literature values are from Delfosse et al. (1998).

In all seven stars, we detected mean magnetic fields of $2 \mathrm{kG}$ or stronger. RB07 investigated $22 \mathrm{M}$ dwarfs, 17 of which have spectral types earlier than M7. All eight of them with normalized $\mathrm{H} \alpha$ luminosity larger than $\log L_{\mathrm{H} \alpha} / L_{\mathrm{bol}}=-4$ also have mean magnetic fields on the order of $2 \mathrm{kG}$ or more. The most active stars in that sample exhibit significant rotation, but the sample contains only one star rotating more rapidly than $v \sin i=10 \mathrm{~km} \mathrm{~s}^{-1}$. Our results are in good agreement with the relation between mean magnetic fields and normalized $\mathrm{H} \alpha$ activity found in RB07. The (projected) rotation velocities of the three most rapidly rotating stars in our sample are at least a factor of two higher, but we see no sign of normalized $\mathrm{H} \alpha$ luminosity higher than in RB07 and no exceptionally high value of $B f$. The main result of this work is that none of the three rapid rotators with projected rotation velocities close to $20 \mathrm{~km} \mathrm{~s}^{-1}$ shows a mean magnetic field above $3 \mathrm{kG}$.

Can we really measure magnetic fields in stars rotating as rapidly as $v \sin i=20 \mathrm{~km} \mathrm{~s}^{-1}$ ? In Reiners \& Basri (2006) we have shown that the magnetic sensitivity of $\mathrm{FeH}$ in principle allows the measurement of magnetic flux in stars rotating as rapidly as $v \sin i=30 \mathrm{~km} \mathrm{~s}^{-1}$. The limiting factor in rapid rotators is the achievable signal-to-noise ratio. In our case of $v \sin i \approx 20 \mathrm{~km} \mathrm{~s}^{-1}$ "only", this is not a crucial problem. The differences between stars with strong and weak magnetic fields in the presence of rapid rotation can be seen in the spectra we show in Figures 3 and 4 . The results of our $\chi^{2}$ fits plotted in the lower panels of Figures 3 and 4 show that in all three stars $\chi^{2}$ becomes significantly larger at very low field strengths or if one allows for mean fields as high as $4 \mathrm{kG}$.

Our active template star Gl 873, which we are using as comparison for our targets, has a mean magnetic field of about $4 \mathrm{kG}$. From comparison to the spectrum of Gl 873, we cannot measure magnetic flux in excess of that value. In RB07 we show a spectrum of YZ CMi (M4.5, $v \sin i=5 \mathrm{~km} \mathrm{~s}^{-1}$ ), from which we inferred a mean field stronger than $4 \mathrm{kG}$. This spectrum shows that in the presence of stronger fields the magnetically sensitive lines can become even more washed out following the principles of Zeeman broadening. ${ }^{6}$ The spectrum of Gl 412 B (WX UMa) shows the same behavior at a rotation velocity of $v \sin i=5 \mathrm{~km} \mathrm{~s}^{-1}$. Thus, although we lack a spectrum independently calibrated to stronger magnetic flux to compare with, we see no way that the spectra of the three most rapidly rotating stars can be consistent with magnetic flux stronger than $B f=4 \mathrm{kG}$. We discuss these stars further below.

\footnotetext{
6 We show this spectrum in Figure 3 of RB07. In that plot, the ratio of the magnetically sensitive to the insensitive lines is smaller in YZ CMi than in our magnetically active template.
}

\subsection{Saturation of the Magnetic Flux Bf}

It is well accepted that coronal and chromospheric activity saturate at high rotation rates. Mohanty \& Basri (2003) showed that this relation is still valid in stars as late as spectral type M8.5. In mid-M stars, all stars with detected rotational broadening show $\mathrm{H} \alpha$ emission at the saturation level. In the left panel of Figure 5, we plot $v \sin i$ versus $\log L_{\mathrm{H} \alpha} / L_{\mathrm{bol}}$ for all stars of spectral types earlier than M7 from RB07, together with the stars from our new sample. This shows that the rotationactivity relation is still valid in the combined sample. In the right panel of Figure 5, we plot measured magnetic flux $B f$ as a function of $v \sin i$. If magnetic flux did not saturate, we would expect the values of $B f$ to continue growing with higher rotation rate. This is not observed: instead, the magnetic flux shows the same saturation effect as $\mathrm{H} \alpha$ emission. From this result we conclude that magnetic flux generation does not grow any further in stars with saturated $\mathrm{H} \alpha$ emission. In other words, magnetic flux saturates in roughly the same fashion as activity, implying that activity does not saturate solely because the whole area of the star is covered with fields $(f=1)$.

\subsection{Comparison with Hotter Stars}

How does the saturation of magnetic flux in our M-dwarf sample fit into the picture of rotation and activity in hotter stars? To compare activity measurements in very different stars, the Rossby number, i.e., the ratio of rotation period $P$ and convective overturn time $\tau_{\text {conv }}$, is the parameter of choice. We note, however, that the main effect of the Rossby number is to compare rotation periods, and that the influence of the convective overturn time is still debatable. Kiraga \& Stępień (2007) provide a measurement of the rotation period of Gl 729. For Gl 873, AD Leo, and YZ CMi, $P$ can be found in Saar (2001). To obtain the (projected) Rossby number, Ro $=P / \tau_{\text {conv }}$, for the other stars, we calculate the (projected) rotation periods for our sample and the stars from RB07 from the projected surface rotation velocity $v \sin i$ for which we require the radii. Note that this only provides $\mathrm{Ro} / \sin i$, which is an upper limit of Ro. To determine the radius, we employed the mass-luminosity relation from Delfosse et al. (2000) and the mass-radius relation at an age of 5 Gyr from Baraffe et al. (1998). To compute the masses, we used Jmagnitudes from Cutri et al. (2003) and distances from Hawley et al. (1996). Parallaxes for Gl 299 and GJ 1286 are from Harrington \& Dahn (1980) and Oppenheimer et al. (2001), respectively. For the convective overturn time, we adopt a value of $\tau_{\text {conv }}=70 \mathrm{~d}$, consistent with the values given for M dwarfs in Saar (2001), which are taken from Gilliland (1986). Kiraga \& Stępień (2007) have recently calculated empirical turnover times for a sample of $\mathrm{M}$ stars. Their relation yields values of $\tau_{\text {conv }} \sim 50-100 \mathrm{~d}$ for the mass range considered in our sample. This is roughly consistent with $\tau_{\text {conv }}=70 \mathrm{~d}$; if we were using the convective overturn times from Kiraga \& Stępień (2007), the Rossby numbers would be about 0.15 dex larger (smaller) for the hottest (coolest) stars. This difference does not affect our conclusions. Radii, (projected) periods, and Rossby numbers are given in Table 3 .

We combine our measurements with data on additional stars from Saar (1996, 2001). For the stars from Saar (1996) we adopt a value of $\tau_{\text {conv }}=20 \mathrm{~d}$ (spectral type G0-K5), noting that the convective overturn time changes over this range of stars but that the difference is not significant on the level considered here.

The behavior of $B f$ with Rossby number is shown in Figure 6, where we plot all the mentioned samples together. Data from this work are plotted as filled circles, data from RB07 as 

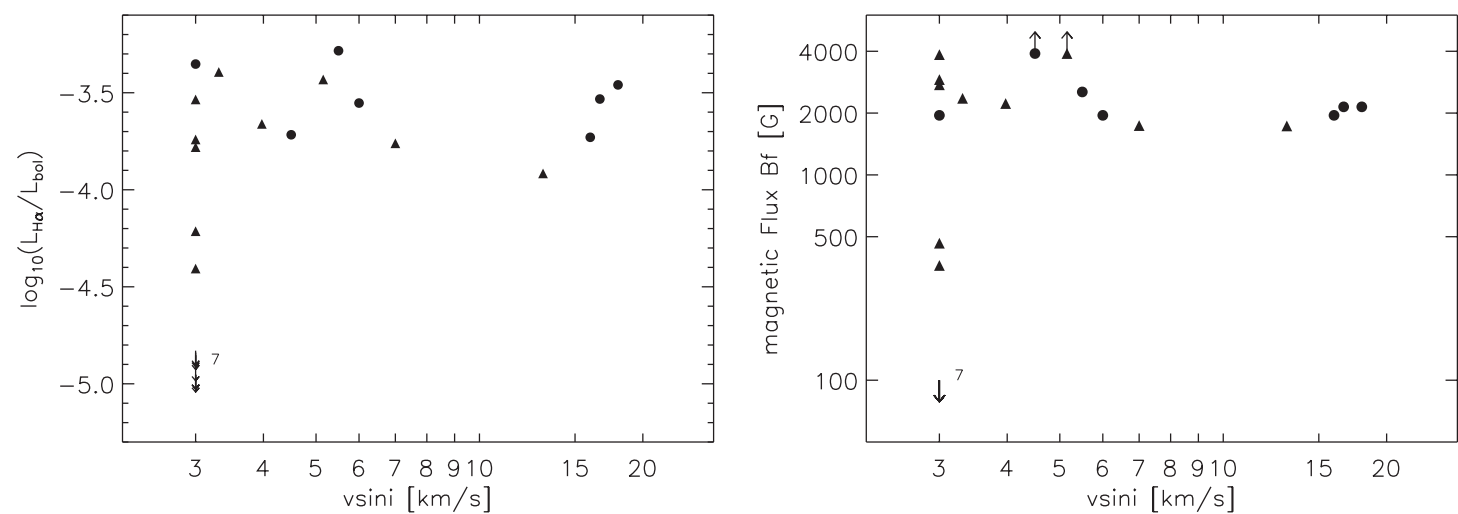

Figure 5. Left panel: normalized $\mathrm{H} \alpha$ activity as a function of $v \sin i$ in $\mathrm{M}$ stars. Right panel: magnetic flux $B f$ as a function of $v \sin i$. Filled circles are from this work, triangles come from RB07. Downward arrows indicate upper limits; numbers give the number of multiple measurements with the same results. The two lower limits of $B f$ are indicated with upward arrows.

Table 3

Properties of Our Sample Stars and the Stars from RB07

\begin{tabular}{lllllrrrrr}
\hline \hline Name & Other & SpT & $M / M_{\odot}$ & $R / R_{\odot}$ & $v \sin i$ & $P / \sin i$ & $\log (\mathrm{Ro} / \sin i)$ & $B f(\mathrm{G})$ & $\log L_{\mathrm{X}} / L_{\text {bol }}$ \\
\hline GJ 3379 & & M3.5 & 0.24 & 0.25 & $<3$ & $>4.2$ & $>-1.2$ & 2000 & -2.86 \\
GJ 2069 B & CV Cnc & M4.0 & 0.24 & 0.25 & 6 & 2.3 & -1.5 & 2500 & -2.77 \\
G1 493.1 & FN Vir & M4.5 & 0.17 & 0.20 & 18 & 0.5 & -2.1 & 2100 & -3.31 \\
LHS 3376 & & M4.5 & 0.14 & 0.17 & 16 & 0.5 & -2.1 & 2000 & -3.63 \\
GJ 1154 A & & M5.0 & 0.18 & 0.20 & 6 & 1.7 & -1.6 & 2000 & -3.28 \\
GJ 1156 & GL Vir & M5.0 & 0.14 & 0.16 & 17 & 0.5 & -2.1 & 2100 & -3.39 \\
G1 412 B & WX UMa & M6.0 & 0.11 & 0.13 & 5 & 1.4 & -1.7 & $>3900$ & -3.28 \\
\hline & & & & \multicolumn{2}{c}{ Stars from RB07 } & & & \\
\hline G1 70 & & M2.0 & 0.35 & 0.33 & $<3$ & $>5.6$ & $>-1.1$ & 0 & $<-4.44$ \\
G1 729 & V1216 Sgr & M3.5 & 0.18 & 0.20 & 4 & 2.9 & -1.4 & 2200 & -3.50 \\
G1 873 & EV Lac & M3.5 & 0.33 & 0.31 & 3 & 4.4 & -1.2 & 3900 & -3.07 \\
G1 388 & AD Leo & M3.5 & 0.42 & 0.39 & 3 & 2.6 & -1.4 & 2900 & -3.02 \\
G1 876 & & M4.0 & 0.32 & 0.31 & $<3$ & $>5.2$ & $>-1.1$ & 0 & -5.23 \\
GJ 1005A & & M4.0 & 0.22 & 0.23 & $<3$ & $>3.9$ & $>-1.3$ & 0 & -5.05 \\
G1 299 & & M4.5 & 0.15 & 0.18 & $<3$ & $>3.0$ & $>-1.4$ & 500 & $<-5.55$ \\
GJ 1227 & & M4.5 & 0.17 & 0.19 & $<3$ & $>3.2$ & $>-1.3$ & 0 & $<-3.86$ \\
GJ 1224 & & M4.5 & 0.15 & 0.18 & $<3$ & $>3.0$ & $>-1.4$ & 2700 & -3.06 \\
G1 285 & YZ CMi & M4.5 & 0.31 & 0.30 & 5 & 2.8 & -1.4 & $>3900$ & -3.02 \\
G1 905 & HH And & M5.0 & 0.14 & 0.17 & $<3$ & $>2.8$ & $>-1.4$ & 0 & -3.75 \\
GJ 1057 & & M5.0 & 0.16 & 0.18 & $<3$ & $>3.1$ & $>-1.4$ & 0 & $<-3.87$ \\
GJ 1245B & & M5.5 & 0.11 & 0.14 & 7 & 1.0 & -1.8 & 1700 & -3.58 \\
GJ 1286 & & M5.5 & 0.12 & 0.14 & $<3$ & $>2.4$ & $>-1.5$ & 400 & $<-3.77$ \\
GJ 1002 & & M5.5 & 0.11 & 0.13 & $<3$ & $>2.3$ & $>-1.5$ & 0 & $<-5.24$ \\
G1 406 & CN Leo & M5.5 & 0.10 & 0.13 & 3 & 1.9 & -1.6 & 2400 & -2.77 \\
GJ 1111 & DX Cnc & M6.0 & 0.10 & 0.12 & 13 & 0.4 & -2.2 & 1700 & -3.88 \\
\hline
\end{tabular}

Notes.

${ }^{a}$ Kiraga \& Stępień (2007).

${ }^{b}$ Saar (2001).

open circles (M dwarfs), and data from Saar (1996, 2001) as crosses (note that crosses are hotter stars). Stars in which only upper limits of $v \sin i$ were measured are not shown (nondetections of rotation). Figure 6 clearly shows the dependence of magnetic flux generation on Rossby number: At large Rossby numbers $($ Ro $>0.1)$, i.e., in the nonsaturated regime, magnetic flux increases with decreasing Ro (more rapid rotation). At smaller Rossby number, i.e., in the regime where $\mathrm{H} \alpha$ and $\mathrm{X}$-ray emission saturate, $B f$ saturates as well.

In Figure 6, the saturated regime mainly consists of $\mathrm{M}$ dwarfs while the rising part of the relation (crosses) is populated by hotter stars. Reiners (2007) showed that a drop in activity with higher Rossby number also appears in slowly rotating $\mathrm{M}$ dwarfs (his Figure 10). A substantial piece of evidence for an intact rotation-activity relation among $\mathrm{M}$ dwarfs can be found in the data presented by Kiraga \& Stępień (2007). From their data, we show normalized X-ray activity versus Rossby number (again assuming $\tau_{\text {conv }}=70 \mathrm{~d}$ ) in Figure 7. Clearly, a rise of activity at high Rossby number $(\mathrm{Ro}=0.1 \ldots 1)$ and a saturation plateau at lower Rossby number appears among $\mathrm{M}$ dwarfs too.

We conclude that the dynamo process saturates at Rossby number of about Ro $=0.1$. No systematic increase of $B f$ occurs if the Rossby number is smaller by another order of magnitude.

\subsection{The Regime of Saturation}

The level of magnetic flux in the saturated regime is between 2 and $4 \mathrm{kG}$. In two stars, we observe magnetic flux that may be higher than $4 \mathrm{kG}$. At least one of the two stars is not among those with the smallest Rossby numbers in our sample. We can speculate whether the Rossby numbers of both stars with the 


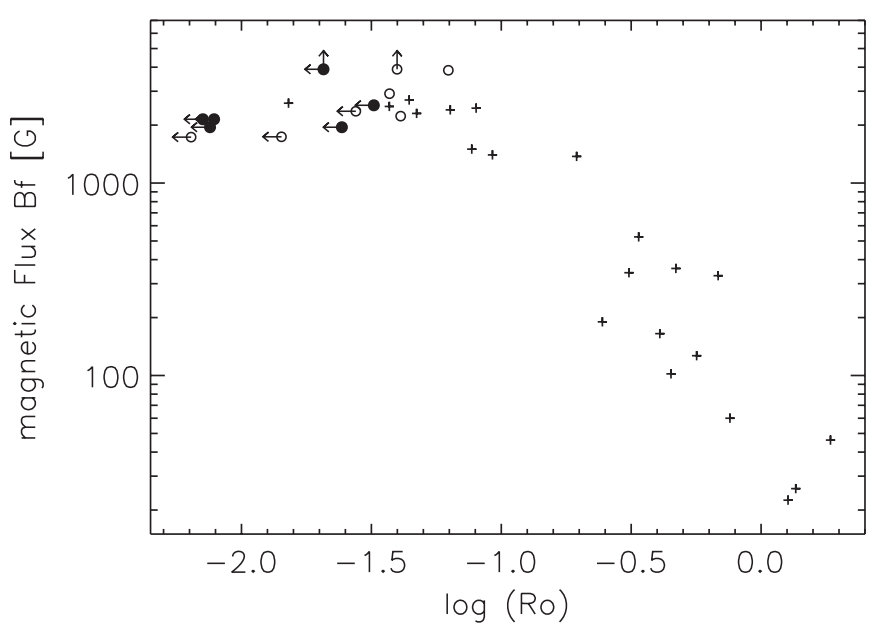

Figure 6. Magnetic flux $B f$ as a function of Rossby number. Data are from Saar (1996, 2001), RB07, and from this work. Eleven stars without measured rotation periods and with no detection of rotational broadening $\left(v \sin i<3 \mathrm{~km} \mathrm{~s}^{-1}\right)$, i.e., lower limits of Ro, are not shown. They would form a vertical line at about $\log$ Ro $=-1$ but probably lie on top of the rising part of the correlation (see Reiners 2007). Data from this work are plotted as filled circles, data from RB07 as open circles (M stars), and data from Saar $(1996,2001)$ as crosses (spectral types G0-M2). Rotation rates from this work and from RB07 are calculated from $v \sin i$ implying that open and filled circles are Ro/ $\sin i$ and are hence upper limits of Ro.

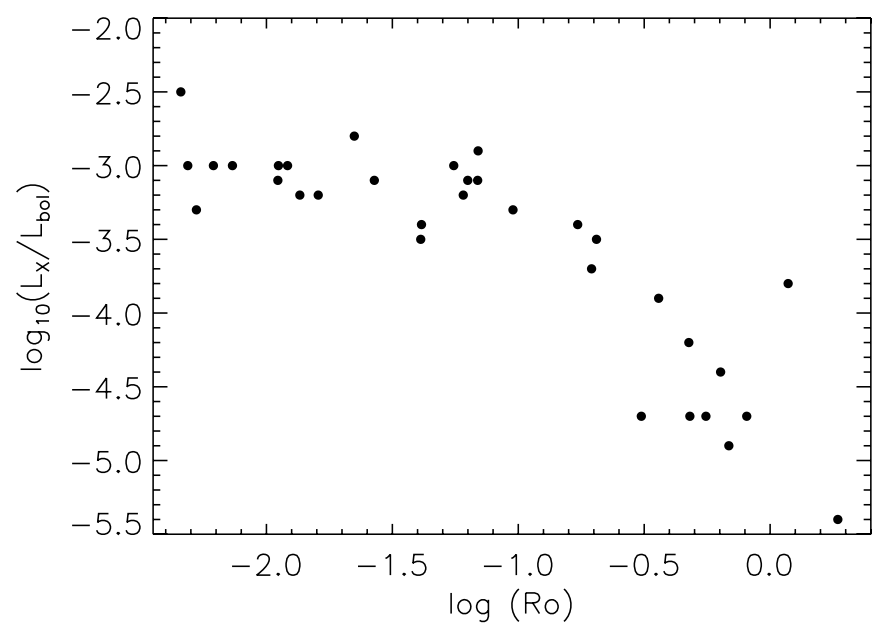

Figure 7. Normalized X-ray activity as a function of Rossby number in M stars (using $\tau_{\text {conv }}=70 \mathrm{~d}$ ) from Kiraga \& Stepien (2007). This plot is essentially the same as their Figure 7 but in logarithmic units, so that the saturation plateau becomes clear.

highest magnetic flux are smaller than the Rossby numbers of the other stars in our sample, i.e., whether the Rossby numbers of YZ CMi and WX UMa are also on the order of $\log$ Ro $\approx-2$. In the case of WX Uma, we only have Ro/ $\sin i$, and for a real value of $\log \mathrm{Ro}=-2$ the star would be observed under an inclination angle of $i<30^{\circ}$. For WX UMa, this is a viable option. On the other hand, a rotation period of $P=2.8 \mathrm{~d}$ is reported for YZ CMi, which is in good agreement with an inclination angle close to $i=90^{\circ}$ given the estimated radius and the measured rotation velocity. To push the value of $\log$ Ro $\approx-1.4$ to -2 , either the rotation velocity must be a factor of four higher ( $i<15^{\circ}$ implying that the rotation period is wrong), or the convective overturn time must be longer by the same amount. Both options seem rather unlikely. We note that Saar (2001) reported a magnetic flux of $B f=3.3 \mathrm{kG}$ for $\mathrm{YZ} \mathrm{CMi}$, a value that is somewhat lower than our result. This may indicate that

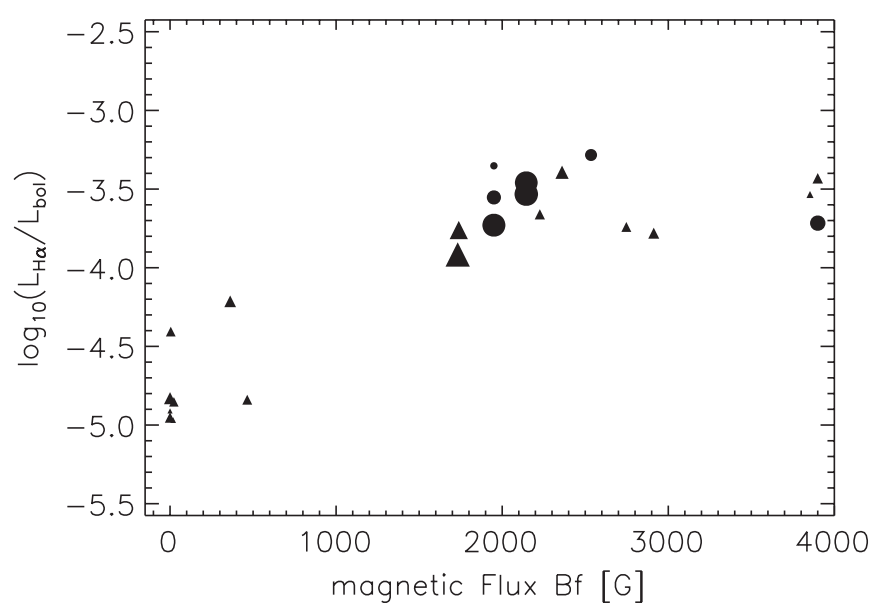

Figure 8. Correlation between $\mathrm{H} \alpha$ activity and magnetic flux $B f$ in $\mathrm{M}$ stars. Symbol size scales with inverse Rossby number (large symbols have small Ro). Triangles are from RB07, circles are from this work.

our value does not reflect an unusually strong average field strength in YZ CMi, but that the magnetic flux shows rather large scatter (either due to uncertainties in the measurements or temporal fluctuations).

The easiest explanation for the two very high values of $B f>4 \mathrm{kG}$ is that the scatter in the saturated magnetic flux level is fairly large, and $B f$ between 2 and $5 \mathrm{kG}$ might just be the allowed range at small Rossby numbers (including observational effects). We have searched for other parameters, such as exceptionally low gravity, that could cause the high flux values in the two stars. We did not find any particular stellar parameter that distingiushes YZ CMi and WX Uma from other flare stars. In particular, age is probably of little direct importance for the generation of very high magnetic flux: WX Uma is an old disk flare star while YZ CMi is a member of the young disk population (Veeder 1974).

Even if the two strongest magnetic flux measurements are due to exceptionally small Rossby numbers, this would not explain the saturation of $B f$ between $\log$ Ro $=-2$ and -1 . Across an order of magnitude in Ro, $B f$ varies by at most a factor of a few. This is in striking contrast to the nearly hundredfold increase in $B f$ in going from Ro $\approx 1$ to 0.1 . We have concluded that both magnetic flux and chromospheric $\mathrm{H} \alpha$ (as well as coronal X-ray) emission saturate at small Ro. With the large uncertainties in $B f$, we cannot reliably determine whether there is any supersaturation effect, with magnetic flux declining in the most rapid rotators.

Although both emission and magnetic flux appear to saturate at small Ro, we observe a large scatter of magnetic flux among the stars with small Ro and likewise some scatter in $\mathrm{H} \alpha$ emission. We thus examined whether a relation between $\mathrm{H} \alpha$ emission and magnetic flux still exists at very small Rossby numbers. In Figure 8 , we plot normalized $\mathrm{H} \alpha$ emission versus magnetic flux for our stars. As expected, $\log L_{\mathrm{H} \alpha} / L_{\text {bol }}$ grows as $B f$ grows from 0 to $2 \mathrm{kG}$. Beyond that, i.e., in the regime of saturated magnetic flux, no further increase in chromospheric emission is observed, although $B f$ grows as large as $4 \mathrm{kG}$. No obvious correlation exists between chromospheric emission and magnetic flux in the regime of saturation. This could mean that saturation of chromospheric emission occurs above a certain level of integrated flux on the star (for example, because the filling factor reached unity). The field could then grow stronger 
without causing further heating. This is in fact not ruled out by our poorly constrained understanding of chromospheric heating mechanisms.

As of now, the available data do not permit us to distinguish reliably between these two possibilities. But, as noted above, the most rapid rotators in our sample probably do not possess magnetic fluxes higher than among other stars in the saturated regime: in Figure 8, stars with the smallest Rossby numbers lie at the low- $B f$ end of the plateau. Neither are they the stars with the highest normalized chromspheric emission. Thus the lack of tight correlation between emission and $B f$ in the saturation regime should not be construed as evidence that magnetic flux continues to be correlated with rotation but decouples from emission. Instead, our data suggest that any variation of $\mathrm{H} \alpha$ emission or $B f$ in the saturation regime must either be randomi.e., due to the observational scatter-or depend on parameters other than rotation. At this stage, we note only that the plateau in Figure 8 is consistent with scatter alone.

\section{SUMMARY}

Using absorption lines of molecular $\mathrm{FeH}$, we have measured the magnetic flux in seven M stars that are known X-ray sources. With one exception, they were known as rapid rotators as well. We reinvestigated the projected rotational velocities $v \sin i$ and measured chromospheric emission in $\mathrm{H} \alpha$. All stars proved to be strong $\mathrm{H} \alpha$ emitters. In our analysis of $v \sin i$, we found some inconsistencies with former literature, which we ascribe to our more sophisticated (direct) fitting method and better data quality.

All of our target stars show strong $\mathrm{H} \alpha$ emission at the saturation level $\left(\log L_{\mathrm{H} \alpha} / L_{\mathrm{bol}}>-4\right)$. We detected magnetic fields of $\mathrm{kG}$ strength in all our targets. While in less rapidly rotating stars $\mathrm{H} \alpha$ and magnetic flux correlate with rotation rate, no such correlation is observed in our sample.

In contrast to Sun-like stars of spectral types G and K, M dwarfs rotating at $v \sin i \approx 10 \mathrm{~km} \mathrm{~s}^{-1}$ have very small Rossby numbers. Thus, our sample targets add to the amount of stars with measured magnetic flux in the regime of small Rossby number, i.e., in the regime of saturated magnetic activity. Our primary goal was to determine whether magnetic flux in this regime continues to grow with more rapid rotation, as sometimes suggested (e.g., Saar 1996), or instead saturates in the same manner as coronal and chromospheric emission.

Our main conclusion is that around a Rossby number of Ro $\sim 0.1$, magnetic flux saturates at approximately $B f=3 \mathrm{kG}$. Below Ro $\sim 0.1, B f$ does not grow stronger with decreasing Rossby number. In looking at the effect of Rossby number (Ro $\left.=P / \tau_{\text {conv }}\right)$, we are primarily sensitive to the effects of rotation, because the sampled rotations vary by a factor of more than 10 while the convective overturn time probably changes less than a factor of two in the range of $\mathrm{M}$ stars we are considering.

In the regime of saturated magnetic flux and chromospheric emission, we still observe a strong scatter in magnetic flux. The interpretation of this feature is not clear. It may be that below the critical Rossby number, normalized $\mathrm{H} \alpha$ emission is not sensitive to changes in $B f$. Alternatively, in light of the large systematic errors of our $B f$ measurements, the scatter of $B f$ in the saturated regime may be fully explained by observational uncertainties.

Our results indicate that the strengths of stellar magnetic fields, and not merely their filling fractions on the surface alone, reach a maximum at a certain rotation rate. The scatter in magnetic flux among the stars with very small Rossby numbers, however, is substantial. Typical values are between 2 and 4 $\mathrm{kG}$. Two stars show magnetic flux stronger than $4 \mathrm{kG}$, and we cannot exclude that individual stars can generate magnetic flux stronger than that. Determining what sets the maximum valuewhether equipartition with either the atmospheric pressure or the turbulent velocity field or some more subtle effect - remains a challenge for future observations and theories.

This work is based on observations from the W.M. Keck Observatory, which is operated as a scientific partnership among the California Institute of Technology, the University of California and the National Aeronautics and Space Administration. We would like to acknowledge the great cultural significance of Mauna Kea for native Hawaiians and express our gratitude for permission to observe from atop this mountain. A.R. acknowledges research funding from the DFG under an Emmy Noether Fellowship (RE 1664/4-1). G.B. acknowledges support from the NSF through grant AST-0606748. M.B. was supported by an NSF Astronomy and Astrophysics postdoctoral fellowship (AST-0502413).

\section{REFERENCES}

Allard, F., Hauschildt, P. H., Alexander, D. R., Tamanai, A., \& Schweitzer, A. 2001, ApJ, 556, 357

Ayres, T. 1999, ApJ, 525, 240

Baraffe, I., Chabrier, G., Allard, F., \& Hauschildt, P. H. 1998, A\&A, 337, 403

Cutri, R. M., et al. 2003, The 2MASS All-Sky Catalog of Point Sources, University of Massachusetts, Infrared Processing and Analysis Center (IPAC) at California Institute of Technology

Delfosse, X., Forveille, T., Perrier, C., \& Mayor, M. 1998, A\&A, 331, 581

Delfosse, X., Forveille, T., Ségransan, D., Beuzit, J.-L., Udry, S., Perrier, C., \& Mayor, M. 2000, A\&A, 364, 217

Harrington, R. S., \& Dahn, C. C. 1980, AJ, 85, 454

Hawley, S. L., Gizis, J. E., \& Reid, I. N. 1996, AJ, 112, 2799

James, D. J., Jardine, M. M., Jeffries, R. D., Randich, S., Collier Cameron, A., \& Ferreira, M. 2000, MNRAS, 318, 1217

Johns-Krull, C., \& Valenti, J. A. 2000, in ASP Conf. Ser. 198, Stellar Clusters and Associations: Convection, Rotation, and Dynamos, ed. R. Pallavicini, G. Micela, \& S. Sciortino (San Francisco: CA: ASP), 371

Gilliland, R. L. 1986, ApJ, 300, 339

Kiraga, M., \& Stępień, K. 2007, AcA, 57, 149

Mohanty, S., \& Basri, G. 2003, ApJ, 583, 451

Noyes, R. W., Hartmann, L. W., Baliunas, S. L., Duncan, D. K., \& Vaughan, A. H. 1984, ApJ, 279, 763

Oppenheimer, B. R., Golimowski, D. A., Kulkarni, S. R., Matthews, K., Nakajima, T., Creech-Eakman, M., \& Durrance, S. T. 2001, AJ, 121, 2189

Pizzolato, N., Maggio, A., Micela, G., Sciortino, S., \& Ventura, P. 2003, A\&A, 397,147

Reiners, A. 2007, A\&A, 467, 259

Reiners, A., \& Basri, G. 2006, ApJ, 644, 497

Reiners, A., \& Basri, G. 2007, ApJ, 656, 1121

Reiners, A., \& Basri, G. 2008, A\&A, 489, L45

Robinson, R. D. 1980, ApJ, 239, 961

Saar, S. H. 1996, in IAU Symp. 176, Stellar Surface Structure, ed. K. G. Linsky \& J. L. Strassmeier (Dordrecht: Kluwer), 237

Saar, S. H. 2001, ASP Conf. Ser. 223, 11th Cambridge Workshop on Cool Stars, Stellar Systems and the Sun (San Francisco: CA: ASP), 292

Schmitt, J. H. M. M., \& Liefke, C. 2004, A\&A, 417, 651

Solanki, S. K. 1994, in ASP Conf. Ser. 64, Structure of the solar chromosphere, ed. A. V. Stepanov, E. E. Benevolenskaya, \& A. G. Kosovichev (San Francisco, CA: ASP), 477

Veeder, G. J. 1974, AJ, 79, 702

Vilhu, O. 1984, A\&A, 133, 117

Vilhu, O., \& Vilhu, O. 1987, in Lecture Notes in Physics, Vol. 291, ed. J. L. Linsky \& R. E. Stencel (Berlin: Springer), 110 